\title{
MtDNA variability in the field vole (Microtus agrestis L., 1761), Arvicolinae, Rodentia) in the Urals and adjacent territories
}

\author{
Maria Krokhaleva \\ Institute of Plant and Animal Ecology \\ UB RAS, \\ Ekaterinburg, Russia \\ krohaleva_ma@ipae.uran.ru \\ Evgenia Markova \\ Institute of Plant and Animal Ecology \\ UB RAS, \\ Ekaterinburg, Russia \\ e.markova@ipae.uran.ru
}

\author{
Lidia Yalkovskaya \\ Institute of Plant and Animal Ecology \\ UB RAS, \\ Ekaterinburg, Russia \\ lida@ipae.uran.ru \\ Aleksandr Borodin \\ Institute of Plant and Animal Ecology \\ UB RAS, \\ Ekaterinburg, Russia \\ bor@ipae.uran.ru
}

\author{
Petr Sibiryakov \\ Institute of Plant and Animal Ecology \\ UB RAS, \\ Ekaterinburg, Russia \\ sibiryakov@ipae.uran.ru
}

\begin{abstract}
The data on mtDNA variability (complete cyt $b$ gene) in M. agrestis in the Cis-Urals, the Southern, Middle, Northern Urals, and Western Siberia obtained for the first time expand significantly the geography of investigations of the species genetic diversity. Analysis of the genetic variability of the field vole with the inclusion of new data does not contradict present views on the genetic structure of the species but reveals genetic heterogeneity of populations within the Eastern subclade, which has not been considered previously. To clarify the genetic structure of the wide-ranging Eastern subclade of $M$. agrestis (from Norway to Lake Baikal), further studies of the species in Northern Eurasia are necessary.
\end{abstract}

Keywords - Microtus agrestis, genetic variability, cytochrome b, Northern Eurasia

\section{Motivation and Aim}

\section{Motivation}

The field vole is a widespread Palearctic species, ranging from Western Europe eastwards to Lake Baikal in southeast Siberia [1]. Due to its wide distribution the species is an excellent model for studying genetic variability. Variation of mtDNA based on cyt $b$ data in $M$. agrestis has been relatively well-studied [2-6]. However, there are almost no data on the central part of Northern Eurasia, namely on the Urals and adjacent areas. Geographical location of the Ural region determines the diversity of its climatic conditions and the uniqueness of its fauna, comprising both the European and Asian elements as well as widespread species of mammal.

Aim

The aim of the study was to analyze mtDNA variability in the field vole in the Urals and adjacent territories based on cyt $b$ data and to determine the position of $M$. agrestis from the studied territory in the phylogeographic structure of the species.

\section{Methods}

Total genomic DNA was isolated by the method of salt extraction from muscle tissue specimens fixed in $96 \%$ ethanol. PCR and subsequent sequencing of the cyt $b$ was carried out with primers L7 and H6. The chromatograms were analyzed using the BioEdit v. 7.2.0 (4.30.2013) software program. Sequence alignments were carried out in MEGA v. 6. Construction of phylogenetic trees using Bayesian inference
(BI) was carried out in MrBayes v. 3.2.2. Assessment of nucleotide diversity was performed in the Arlequin v. 3.1 and DnaSP v. 5.10 software programs.

\section{Results}

In order to analyze mtDNA variability, complete cyt b (1140 bp) is sequenced from 33 samples of M. agrestis from 13 localities in the Cis-Urals, the Southern, Middle, Northern Urals, and Western Siberia. 23 haplotypes are identified, all of them are original and have not been described previously. For comparative analysis, a total of 373 haplotypes of $M$. agrestis are used including our data and 350 haplotypes from GenBank. $M$. arvalis $(\mathrm{n}=2), M$. rossiaemeridionalis $(\mathrm{n}=2), M$. socialis $(\mathrm{n}=2), M$. kirgisorum $(\mathrm{n}=2)$ are used as an out-group.

Based on phylogenetic tree constructed using Bayesian inference (BI) three main clades are recognized: South, Portugal, and Northern clade. The Northern clade is widespread and differentiated into 6 subclades. Newly obtained results correspond with the data from previous studies $[4,6]$. All haplotypes sequenced for M. agrestis in the Urals and adjacent territories are included in the Eastern subclade of the Northern clade.

The Eastern subclade differentiation has not been previously considered despite the significant area of its distribution (from Norway to the eastern boundary of the species range), including regions with different physical, geographical and environmental conditions, geological history. Analysis of the genetic variability of the species with the inclusion of new data from the Cis-Urals, Urals and Western Siberia indicates genetic heterogeneity within the Eastern haplogroup of the field vole. The two major haplogroups are revealed in the Eastern subclade of the Northern clade (BI not less than 0,70). One haplogroup (Northern European group) includes haplotypes from Norway, Finland, and Karelia, and another haplogroup (UralWestern Siberia group) includes haplotypes from the Urals and Western Siberia. Nucleotide diversity obtained for these two haplogroups suggests their recent expansion.

The data on mtDNA variability (complete cyt $b$ gene) in M. agrestis in the Cis-Urals, the Southern, Middle, Northern Urals, and Western Siberia obtained for the first time expand significantly the geography of investigations of the species genetic diversity. Analysis of the genetic variability of the 
field vole with the inclusion of new data does not contradict present views on the genetic structure of the species, but reveals the genetic heterogeneity of populations within the Eastern subclade, which was not previously considered. To clarify the genetic structure of $M$. agrestis within the wideranging Eastern subclade (from Norway to Lake Baikal), further studies of the species in Northern Eurasia are necessary.

\section{ACKNOWLEDGMENT}

This study was supported by the Russian Foundation for Basic Research (grant No. 19-04-00966).

\section{REFERENCES}

[1] G. I. Shenbrot and B. R. Krasnov. "Atlas of the geographic distribution of the arvicoline rodents of the world (Rodentia, Muridae: Arvicolinae)," Pensoft, 2005
[2] M. Jaarola and J. B. Searle, "Phylogeography of field voles (Microtus agrestis) in Eurasia inferred from mitochondrial DNA sequences," Molecular ecology, vol. 11, pp. 2613-2621, 2002.

[3] M. Jaarola and J. B. Searle, "A highly divergent mitochondrial DNA lineage of Microtus agrestis in southern Europe," Heredity, vol. 92, pp. 228-234, 2004.

[4] J. S. Herman and J. B. Searle, "Post-glacial partitioning of mitochondrial genetic variation in the field vole," Proceedings of the Royal Society B: Biological Sciences, vol. 278, pp. 3601-3607, 2011.

[5] J. Paupério, J. S. Herman, J. Melo-Ferreira, M. Jaarola, P. C. Alves, and J. B. Searle, "Cryptic speciation in the field vole: a multilocus approach confirms three highly divergent lineages in Eurasia," Molecular Ecology, vol. 21, pp. 6015-6032, 2012.

[6] J. S. Herman, A. D. McDevitt, A. Kawałko, M. Jaarola, J. M. Wójcik, and J. B. Searle, "Land-bridge calibration of molecular clocks and the post-glacial colonization of Scandinavia by the Eurasian field vole Microtus agrestis," PLoS One, vol. 9, 2014. 